\title{
Measuring the Effect of Blended Learning: Evidence from a Selective Liberal Arts College
}

\author{
Aaron Swoboda and Lauren Feiler \\ Online Appendix
}

\section{Complete Regression Results}

Due to space constraints, we did not include all of the regression covariates in the manuscript. We show the full table of results below.

\section{Additional Variable Descriptions}

Carleton College allows students to report either SAT or ACT scores in their admission file. For comparability, we created a Standardized Test Percentile variable by converting test scores into percentiles and taking the mean percentile across the different reported standardized tests for each student. Percentile data was obtained from https://secure-media.collegeboard.org/ digitalServices/pdf/sat/sat-percentile-ranks-crit-reading-math-writing-2014. pdf and http://www.actstudent.org/scores/norms1.html.

We also collected a financial need variable from our Admissions Office. Each student is designated as one of five need categories. The highest need group was determined to have financial need greater than three-quarters of the comprehensive fee at Carleton College ( $\$ 60,102$ for the 2014-15 academic year). The second highest need group had need greater than half of the comprehensive fee. The third (fourth) highest need categories had need greater than one fourth (zero) of the comprehensive fee. The final group was determined to have no financial need or did not apply for aid. Financial need is bimodally distributed. Approximately half of students received no financial aid, while one quarter of the students were in the highest need category (the remaining quarter of students is distributed across the remaining three aid categories).

\section{Covariates by Subgroups}

Because our study is observational, we tested for differences in student characteristics across the control and treatment class sections (Tables 2 and 3 ) as well as those students who opted out of the study vs those that participated (Tables 4 and 5). No statistically significant differences were found across these groups with the exception of participation by gender. Males were significantly more likely to opt out of the study than females. 
Table 1: Full Regression Results

\begin{tabular}{|c|c|c|c|c|c|c|}
\hline & \multicolumn{6}{|c|}{ Dependent variable: } \\
\hline & \multicolumn{3}{|c|}{$\Delta \mathrm{TUCE}=$ Posttest - Pretest } & \multicolumn{3}{|c|}{$\Delta \mathrm{TUCE} /(30$ - Pretest $)$} \\
\hline & $(1)$ & $(2)$ & $(3)$ & $(4)$ & $(5)$ & $(6)$ \\
\hline Blended Treatment: Yes & $\begin{array}{l}1.92^{* * *} \\
(0.62)\end{array}$ & $\begin{array}{l}1.74^{* * *} \\
(0.61)\end{array}$ & $\begin{array}{l}1.82^{* * *} \\
(0.64)\end{array}$ & $\begin{array}{l}0.09^{* *} \\
(0.04)\end{array}$ & $\begin{array}{c}0.08^{*} \\
(0.04)\end{array}$ & $\begin{array}{c}0.08^{*} \\
(0.04)\end{array}$ \\
\hline Difference in Effort & & $\begin{array}{c}0.06 \\
(0.10)\end{array}$ & $\begin{array}{c}0.09 \\
(0.10)\end{array}$ & & $\begin{array}{c}0.003 \\
(0.01)\end{array}$ & $\begin{array}{c}0.01 \\
(0.01)\end{array}$ \\
\hline Difference in Importance & & $\begin{array}{c}0.17^{*} \\
(0.09)\end{array}$ & $\begin{array}{c}0.12 \\
(0.09)\end{array}$ & & $\begin{array}{c}0.01 \\
(0.01)\end{array}$ & $\begin{array}{c}0.01 \\
(0.01)\end{array}$ \\
\hline Completed College Macro & & & $\begin{array}{c}-0.12 \\
(0.69)\end{array}$ & & & $\begin{array}{c}-0.01 \\
(0.05)\end{array}$ \\
\hline Completed HS Macro & & & $\begin{array}{c}-0.10 \\
(1.04)\end{array}$ & & & $\begin{array}{c}0.05 \\
(0.07)\end{array}$ \\
\hline Completed HS Micro & & & $\begin{array}{c}-0.59 \\
(1.33)\end{array}$ & & & $\begin{array}{c}-0.003 \\
(0.09)\end{array}$ \\
\hline Stand. Test Percentile & & & $\begin{array}{c}-0.07 \\
(0.05)\end{array}$ & & & $\begin{array}{c}0.0001 \\
(0.003)\end{array}$ \\
\hline Year of College & & & $\begin{array}{c}0.05 \\
(0.38)\end{array}$ & & & $\begin{array}{c}-0.02 \\
(0.03)\end{array}$ \\
\hline Male: Yes & & & $\begin{array}{c}-0.69 \\
(0.63)\end{array}$ & & & $\begin{array}{c}-0.04 \\
(0.04)\end{array}$ \\
\hline Minority or Int'l Student & & & $\begin{array}{c}-1.10 \\
(0.70)\end{array}$ & & & $\begin{array}{r}-0.08^{*} \\
(0.05)\end{array}$ \\
\hline Financial Need: Low & & & $\begin{array}{c}0.30 \\
(1.37)\end{array}$ & & & $\begin{array}{c}-0.01 \\
(0.09)\end{array}$ \\
\hline Financial Need: Some & & & $\begin{array}{c}-1.69 \\
(1.07)\end{array}$ & & & $\begin{array}{c}-0.11 \\
(0.07)\end{array}$ \\
\hline Financial Need: More & & & $\begin{array}{c}-2.31^{* *} \\
(1.02)\end{array}$ & & & $\begin{array}{c}-0.15^{* *} \\
(0.07)\end{array}$ \\
\hline Financial Need: Most & & & $\begin{array}{c}-0.84 \\
(0.78)\end{array}$ & & & $\begin{array}{c}-0.04 \\
(0.05)\end{array}$ \\
\hline Constant & $\begin{array}{l}4.34^{* * *} \\
(0.49)\end{array}$ & $\begin{array}{l}4.34^{* * *} \\
(0.48)\end{array}$ & $\begin{array}{l}11.38 \\
(7.92)\end{array}$ & $\begin{array}{l}0.31^{* * *} \\
(0.03)\end{array}$ & $\begin{array}{l}0.31^{* * *} \\
(0.03)\end{array}$ & $\begin{array}{c}0.75 \\
(0.54)\end{array}$ \\
\hline Observations & 150 & 149 & 149 & 150 & 149 & 149 \\
\hline $\mathrm{R}^{2}$ & 0.06 & 0.09 & 0.18 & 0.03 & 0.05 & 0.13 \\
\hline Adjusted $\mathrm{R}^{2}$ & 0.05 & 0.07 & 0.09 & 0.02 & 0.03 & 0.04 \\
\hline
\end{tabular}


Table 2: Demographic Statistics by Class Sections

\begin{tabular}{lcrrrrrr}
\hline \hline \multicolumn{1}{c}{ variable } & group & $\mathrm{n}$ & mean & sd & median & min & max \\
\hline \multirow{2}{*}{ Standardized Test Percentile } & Control & 91 & 94.1 & 6.6 & 95.5 & 57 & 99 \\
& Treatment & 102 & 93.1 & 7.3 & 95.8 & 56 & 99 \\
SAT Math & Control & 54 & 727.4 & 58.0 & 740 & 600 & 800 \\
& Treatment & 71 & 713.2 & 65.5 & 720 & 500 & 800 \\
SAT Verbal & Control & 54 & 696.1 & 70.8 & 695 & 400 & 800 \\
& Treatment & 71 & 694.2 & 67.5 & 700 & 500 & 800 \\
ACT Composite & Control & 49 & 31.6 & 2.6 & 32 & 23 & 36 \\
& Treatment & 52 & 31 & 2.8 & 31.5 & 21 & 35 \\
HS Macro (Yes =1) & Control & 91 & 0.1 & 0.4 & 0 & 0 & 1 \\
\multirow{2}{*}{ HS Micro (Yes =1) } & Treatment & 102 & 0.1 & 0.3 & 0 & 0 & 1 \\
& Control & 91 & 0.1 & 0.3 & 0 & 0 & 1 \\
College Macro (Yes $=1)$ & Treatment & 102 & 0.1 & 0.2 & 0 & 0 & 1 \\
\multirow{2}{*}{ Exp. College Grad Year } & Control & 91 & 0.4 & 0.5 & 0 & 0 & 1 \\
& Treatment & 102 & 0.4 & 0.5 & 0 & 0 & 1 \\
Gender (Male = 1) & Control & 91 & 17.5 & 0.8 & 18 & 15 & 18 \\
& Treatment & 102 & 17.4 & 0.9 & 18 & 15 & 19 \\
Minority or Intl Student & Control & 91 & 0.6 & 0.5 & 1 & 0 & 1 \\
& Treatment & 102 & 0.6 & 0.5 & 1 & 0 & 1 \\
& Control & 91 & 0.4 & 0.5 & 0 & 0 & 1 \\
& Treatment & 102 & 0.3 & 0.5 & 0 & 0 & 1 \\
\hline
\end{tabular}


Table 3: Frequency of Student Financial Need by Class Section

\begin{tabular}{lccccc}
\hline & No Need & Lowest Need & Some Need & More Need & Most Need \\
\hline Control Sections & 42 & 7 & 6 & 13 & 23 \\
Treatment Sections & 51 & 2 & 11 & 11 & 27 \\
\hline
\end{tabular}

Table 4: Student Demographic Statistics by Participation Decision

\begin{tabular}{lcrrrrrr}
\hline \hline \multicolumn{1}{c}{ variable } & group & $\mathrm{n}$ & mean & sd & median & min & max \\
\hline \multirow{2}{*}{ Standardized Test Percentile } & In Study & 150 & 93.3 & 6.8 & 95.5 & 57 & 99 \\
& Opted Out & 43 & 94.4 & 7.4 & 96.5 & 56 & 99 \\
SAT Math & In Study & 104 & 718.5 & 64.3 & 740 & 500 & 800 \\
& Opted Out & 21 & 723.8 & 54.2 & 720 & 600 & 800 \\
SAT Verbal & In Study & 104 & 694.7 & 69.2 & 705 & 400 & 800 \\
& Opted Out & 21 & 696.7 & 67.2 & 690 & 570 & 800 \\
ACT Composite & In Study & 72 & 31.1 & 2.7 & 31 & 23 & 36 \\
& Opted Out & 29 & 31.8 & 2.7 & 32 & 21 & 35 \\
HS Macro (Yes =1) & In Study & 150 & 0.1 & 0.3 & 0 & 0 & 1 \\
\multirow{3}{*}{ HS Micro (Yes =1) } & Opted Out & 43 & 0.1 & 0.4 & 0 & 0 & 1 \\
& In Study & 150 & 0.1 & 0.3 & 0 & 0 & 1 \\
College Macro (Yes $=1)$ & Opted Out & 43 & 0.1 & 0.3 & 0 & 0 & 1 \\
\multirow{2}{*}{ Exp. College Grad Year } & In Study & 150 & 0.4 & 0.5 & 0 & 0 & 1 \\
& Opted Out & 43 & 0.5 & 0.5 & 1 & 0 & 1 \\
Gender (Male = 1) & In Study & 150 & 17.4 & 0.9 & 18 & 15 & 18 \\
& Opted Out & 43 & 17.4 & 0.9 & 18 & 15 & 19 \\
Minority or Intl Student & In Study & 150 & 0.5 & 0.5 & 1 & 0 & 1 \\
& Opted Out & 43 & 0.8 & 0.4 & 1 & 0 & 1 \\
& In Study & 150 & 0.4 & 0.5 & 0 & 0 & 1 \\
& Opted Out & 43 & 0.3 & 0.4 & 0 & 0 & 1 \\
\hline
\end{tabular}

Table 5: Frequency of Student Financial Need by Study Participation Decision

\begin{tabular}{lrrrrr}
\hline & No Need & Low Need & Some Need & More Need & Most Need \\
\hline In Study & 73 & 8 & 14 & 17 & 38 \\
Opted Out & 20 & 1 & 3 & 7 & 12 \\
\hline
\end{tabular}

